Erratum

\title{
MiR-487a Promotes TGF- $\beta 1$-induced EMT, the Migration and Invasion of Breast Cancer Cells by Directly Targeting MAGI2
}

Mengtao Ma1\#, Miao He#, Qian Jiang1, Yuanyuan Yan¹, Shu Guan², Jing Zhang1, Zhaojin Yu ${ }^{1}$, Qiuchen

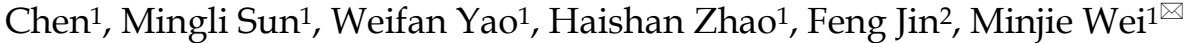

1. Department of Pharmacology, School of Pharmacy, China Medical University, Shenyang, Liaoning Province, China

2. Department of Surgical Oncology, The First Affiliated Hospital of China Medical University, Shenyang, Liaoning Province, China

\#These authors contributed equally to this work.

$\bowtie$ Corresponding author: weiminjiecmu@163.com (Minjie Wei)

(C) The author(s). This is an open access article distributed under the terms of the Creative Commons Attribution License (https://creativecommons.org/licenses/by/4.0/). See http:/ /ivyspring.com/terms for full terms and conditions.

Published: 2021.09.21

Corrected article: Int J Biol Sci 2016; 12(4): 397-408. doi: 10.7150/ijbs.13475.

In our paper [1], Figure 3 should be corrected as follows. 
A
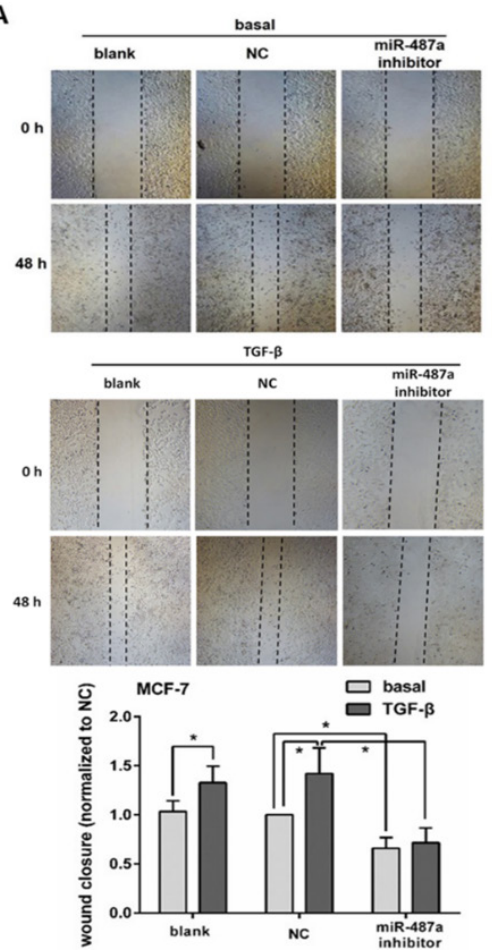

C
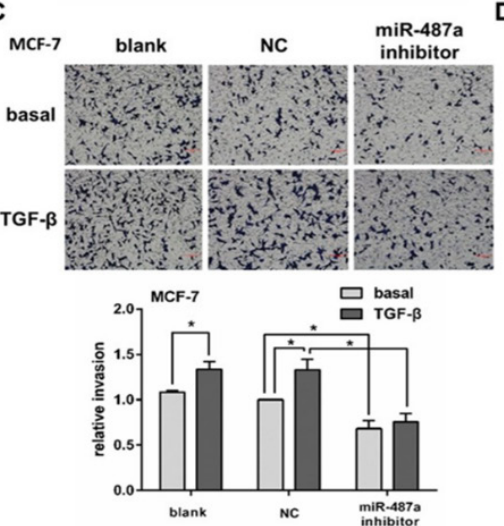

B
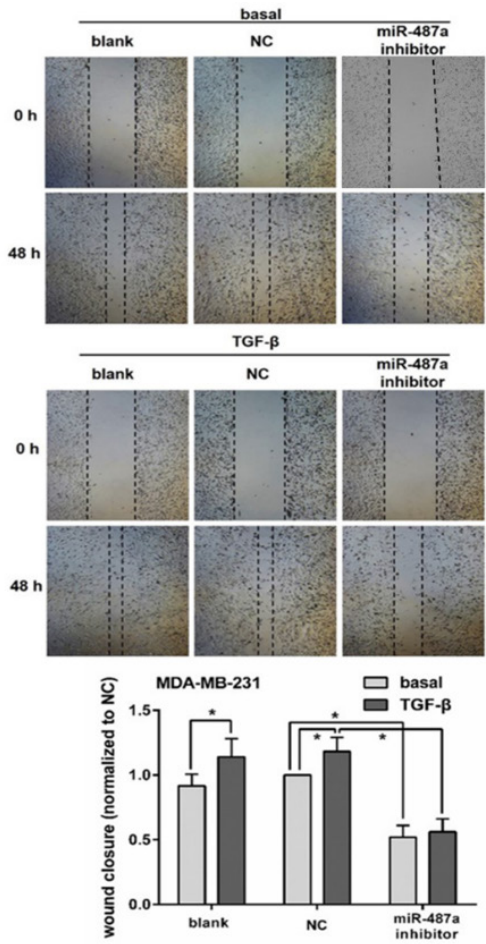

D
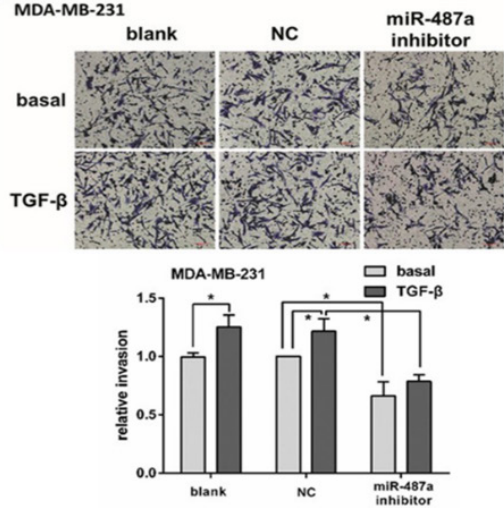

Figure 3. The down-regulation of miR-487a inhibits the migration and invasion induced by TGF- $\beta 1$ in breast cancer cells. The migration abilities were measured by wound healing analysis in MCF-7 cells (A) and MDA-MB-231 cells (B) transfected with miR-487a inhibitor or NC, and treated with or without TGF- $\beta 1$. The wound closure in the cells transfected with NC and treated without TGF- $\beta 1$ was set 1 . The invasion abilities were measured by transwell invasion assay in MCF-7 cells (C) and MDA-MB-231 cells (D) transfected with miR-487a inhibitor or NC, and treated with or without TGF- $\beta 1$. The invasion ability of the cells transfected with NC and treated without TGF- $\beta 1$ was set $1 .{ }^{*} \mathrm{P}<0.05$

\section{References}

1. Mengtao Ma, Miao He, Qian Jiang, Yuanyuan Yan, Shu Guan, Jing Zhang, Zhaojin Yu, Qiuchen Chen, Mingli Sun, Weifan Yao, Haishan Zhao, Feng Jin, Minjie Wei: MiR-487a promotes TGF- 31 -induced EMT, the migration and invasion of breast cancer cells by directly targeting MAGI2. Int J Biol Sci 2016, 12(4):397-408. doi: 10.7150/ijbs.13475. 